Bar-like S-cone stimuli reveal the importance of an intermediate temporal filter

Baraas, Rigmor C. and Kulikowski, Janus J. and Muldoon, Mark R.

2010

MIMS EPrint: 2009.101

Manchester Institute for Mathematical Sciences

School of Mathematics

The University of Manchester

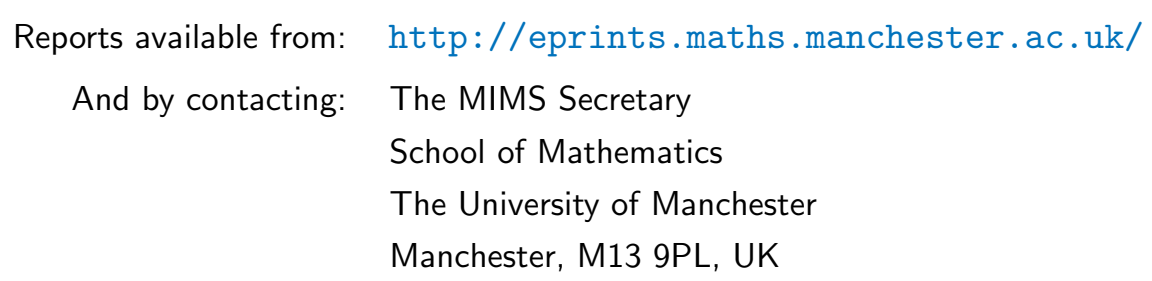

ISSN 1749-9097 


\title{
Bar-like S-cone stimuli reveals the importance of an intermediate temporal filter
}

\author{
Rigmor C. Baraas ${ }^{1, *}$, Janus J. Kulikowski ${ }^{2}$, and Mark R. Muldoon ${ }^{3}$ \\ ${ }^{1}$ Department of Optometry and Visual Science, Buskerud University College, 3611 \\ Kongsberg, Norway \\ ${ }^{2}$ Faculty of Life Sciences, University of Manchester, Manchester M60 1QD, UK \\ ${ }^{3}$ Faculty of Engineering and Physical Sciences, School of Mathematics, University of \\ Manchester, Manchester M13 9PL, UK
}

* Corresponding author: rigmor.baraas@hibu.no 
The relative involvement of different temporal frequency-selective filters underlying detection of chromatic stimuli were studied. Diverse spectral stimuli were used, namely flashed blue and yellow light spots, wide bars and narrow bars. The stimuli were temporally modulated in luminance having constant wavelength. Although the bar-like stimuli apparently reduced the sensitivity at short and long wavelengths, the cone-opponent mechanism still remained responsible for the actual stimulus detection at different temporal frequencies. The bar-like stimuli increased sensitivity for temporal frequencies around 3-6 Hz, revealing involvement of an intermediate temporal frequencyselective filter to detection, the so-called transient-1 filter. A probability summation model for the method of adjustment was developed that assumes that detection depends on the properties of the temporal filters underlying the temporal frequency-sensitivity curve. The model supports the notion that at least two temporal frequency-selective filters are necessary to account for the shape of the sensitivity curves obtained for blue bar-like stimuli. (C) 2010 Optical Society of America

OCIS codes: $330.1690,330.1720,330.6180,330.6790,330.5510$ 


\section{Introduction}

It is now generally thought that three overlapping temporal frequency-selective filters subserve perception of achromatic stimuli [1-5]. These three temporal filters (or channels) can be revealed using a detection task combined with a task of identification of a stimulus, namely detection of pattern, movement and pure flicker $[1,6]$ and corresponds to the three perceptual phenomena [7]. Mandler and Makous [2] also reported three achromatic temporal frequency filters: one low-pass, often referred to as sustained (actually weak band-pass, peak around 0.6-1 Hz), one intermediate band-pass referred to as transient-1 (peak around 3-8 Hz), and a second band-pass referred to as transient-2 (peak 9-16 Hz). The temporal frequencies at which the peaks occur depend on mean luminance, i.e. higher frequencies at higher luminance. The broad tuning of these filters makes it generally difficult to separate them, unless stimuli are chosen to favor certain components, or observers are made to perform discrimination tasks. The peak of the transient-1 temporal filter is most readily

revealed in luminance modulated spatial patterns such as gratings [6,8]. Kelly [8], whilst not recognizing separate filters, observed that luminance modulation is sensitized for frequencies around $5 \mathrm{~Hz}$, following introduction of a bipartite field (a central edge). He also reported that adding a central edge to a chromatic stimulus lowered maximum sensitivity of the low-pass filter. Accordingly, two temporal filters for red-green grating stimuli have been explicitly reported using Gabor patches [9,10]; a low-pass filter (sustained), and a second band-pass filter (transient-2). There are strong residual achromatic contributions due to the L- and M-cone 
phase shifts for red-green stimuli $[11,12]$, and this makes it more difficult to separate either of them from the luminance mechanism. Hence, the intermediate band-pass temporal filter (transient-1) has never been proposed as underlying detection of diffuse chromatic spots. Scone stimuli, like blue presented on a yellow adapting background, give better separation of the cone-opponent mechanisms from the luminance mechanism than L- and M-cone stimuli. A diffuse blue spot on a yellow background would be expected to reveal a broad low-pass temporal frequency-selective filter (sustained), reflecting a pure color mechanism (a color mechanism with balanced red-green and blue-yellow sensitivity). The idea put forward here is that the presence of spatial edges is needed to reveal the intermediate band-pass temporal filter (transient-1). Any evidence of involvement of temporal filters other than the low-pass (sustained) filter would then be taken as evidence for multiple temporal filters subserving chromatic perception in general.

The present study employed rectangular stimuli like bars to investigate the number and type of temporal filters needed to account for detection of blue-yellow stimuli. This was done in an attempt to address the question whether the intermediate band-pass temporal frequency-selective filter (transient-1) is needed in addition to the low-pass temporal frequency-selective filter (sustained) to subserve chromatic processing in general. The addition of high-spatial frequency components, like edges, to S-cone stimuli still left the stimuli predominantly cone-opponent. Thresholds were measured as a function of temporal frequency for short (blue) and medium (yellow) wavelength and it is demonstrated that there is increased sensitivity around 3-8 $\mathrm{Hz}$ for the S-cone bar-like stimuli, suggesting involve- 
ment of the transient-1 filter. A probability summation model was developed and fitted to the experimental data and shows that at least two temporal filters are needed to account for the results. That is: a low-pass filter (sustained), and an intermediate band-pass filter (transient-1), and in some cases a second band-pass filter (transient-2).

The intermediate temporal filter (transient-1) is considered to subserver perception of both color and luminance contrast, but color contrast, and not luminance contrast, is believed to be responsible for detection of blue stimuli for the following reasons:

i) Sensitivity to color contrast is much higher than that for luminance contrast in endspectral regions.

ii) The intermediate temporal filter is also evident when the L-M cone-opponent mechanism has been additionally desensitized with an adapting yellow background.

\section{Methods}

The stimuli were generated by a three-filter Maxwellian view optical system. All stimulus parameters (spatio-temporal and chromatic) as well as observer responses were controlled and analyzed by a computer. The set-up has been described in detail elsewhere [13].

\section{A. Stimuli and backgrounds}

The test stimuli were circular spots $(1 \mathrm{deg})$, wide $(0.5 \times 3.0 \mathrm{deg})$ or narrow bars $(0.06 \mathrm{x}$ $1.3 \mathrm{deg}$ ). The width of the bars subtending 0.06 and 0.5 deg approximates half a cycle of a grating of about 8 and $1 \mathrm{c} / \mathrm{deg}$, respectively. The background of luminous intensity $1000 \mathrm{td}$ 
was neutral daylight $(\mathrm{x}=0.42, \mathrm{y}=0.42$, equivalent to a color temperature $(\mathrm{CT})$ of 3350 $\mathrm{K})$, here referred to as white, or yellow $(\mathrm{x}=0.51, \mathrm{y}=0.47, \mathrm{CT}=2520 \mathrm{~K})$. The yellow background was obtained by adding a Lee industrial filter No.104 to the background field (x $=0.49, \mathrm{y}=0.49)$. The yellow background was used to desensitize the L-M cone-opponent mechanisms, and to facilitate detection of luminance contrast around $575 \mathrm{~nm}[14,15]$.

Calibration of stimuli and backgrounds have been described in detail elsewhere [16,17].

\section{B. Procedures}

In each experiment, the observer used an analogue control button to adjust the intensity of the test object. The stimulus was flickering continuously while the observers were instructed to adjust the intensity until the flicker just ceased to be seen and then made one step up to yield the threshold [18]. Thus these are thresholds for seeing flicker, not any other aspect of a stimulus. Moreover, subjects were encouraged to look away every few seconds to avoid adaptation to flicker. The method is less time consuming [19] and gave similar results as compared with an alternative forced choice method [16] for experienced observers. For slow $(1,2,2.5,3$ and $5 \mathrm{~Hz})$ temporal presentations the observer was instructed to set the threshold for detecting the offset of the test object (seeing the blank between presentations). For rapidly flickering $(7.5,10,15$ and $25 \mathrm{~Hz})$ presentations, the observer was instructed to set the threshold for the detection of flicker [1,6], as during homochromatic flicker photometry [20]. In all experiments, the observer was first dark adapted for 20 min and then adapted to the background for a period of one min before the trials commenced. Fixation was maintained 
central throughout (assisted by a fixation spot).

Two different experiments were carried out. In the first experiment spectral sensitivity curves were measured for 1-deg spots, wide and narrow bars. Measurements of the eleven different wavelength presentations, ranging from 400 to $650 \mathrm{~nm}$ were repeated at least three times. Temporal modulation was 1 or $25 \mathrm{~Hz}$ square-wave with an additive non-zero base. In the second experiment, temporal sensitivity measurements were carried out for two different wavelengths (450 nm, $574 \mathrm{~nm}$ ) with the 1-deg spots, wide and narrow bars. Measurements of the two wavelengths were repeated at least three times and in random order. Temporal modulation was $1,2,2.5,3,5,7.5,10,15$ and $25 \mathrm{~Hz}$ square-wave.

The data presented were obtained from three experienced observers: a 28-year-old female (RB), a 33-year-old male (AG) and a 62-year-old male (JK). AG did not complete the full set of experiments. All observers had corrected monocular visual acuity of $6 / 6$ or better, normal color vision tested with the Farnsworth-Munsell 100 Hue test, and no known ocular or general pathology that could have affected the results.

\section{Model}

\section{A. Probability summation for the method of adjustment}

Following Metha and Mullen [9], it was sought to characterize the observers' sensitivity curves in terms of Watson's probability summation model [21] and various combinations of filters drawn from a family of filters originally proposed by Koenderink [22]. The main idea required to generalize the probability summation model to experiments employing the 
method of adjustment, rather than a two-alternative forced-choice design based on stimuli of fixed temporal duration, is explained briefly below. This is followed by models fitted to the actual data and results. Mathematical details appear in the Appendix, Section A.

\section{B. The probability of recording a threshold at contrast level $C$}

The main result in Section $\mathrm{A}$ is an expression, Eqn. (A9), for $P(C, \nu: \tau, \sigma, \beta, \boldsymbol{A})$, the probability that a stimulus with contrast $C$ and flicker frequency $\nu$ will not be detected by a system of filters specified by parameters $\tau, \sigma$ (which characterize, respectively, the rise-time and temporal duration of the impulse response functions); Weibull parameter $\beta$ and three filter amplitudes given by the vector $\boldsymbol{A}=\left(A_{0}, A_{1}, A_{2}\right)$. These scale, respectively, the output of a low-pass filter $h_{0}$ and two band-pass filters, $h_{1}$ and $h_{2}$ (see Table 1 and Section A for details).

A threshold at contrast level $C$ was recorded if:

i) the stimulus is visible at contrast level $C$, but ...

ii) the stimulus is not visible at contrast level $\gamma C$, where $\gamma=10^{-\epsilon / 10}<1$ and $\epsilon$ (in $\mathrm{dB}$ ), is the smallest step in contrast that the viewing system permits: here $\epsilon=0.3$.

In light of this design, the probability of recording a detection threshold $C$ for a stimulus with flicker frequency $\nu$ is the product

$$
(1-P(C, \nu: \tau, \sigma, \beta, \boldsymbol{A})) \times P(\gamma C, \nu: \tau, \sigma, \beta, \boldsymbol{A})
$$

where the first factor is the probability that the stimulus is detected at contrast $C$ while the 
second is the probability that it is not detected at contrast $\gamma C$.

\section{Bayesian model fitting}

The model builds upon Metha and Mullen's work in two ways, firstly by extending their models to encompass the method of adjustment and secondly by adopting a Bayesian framework for model fitting. The Bayesian approach requires the specification of a prior distribution $P(\tau, \sigma, \beta, \boldsymbol{A})$ on the parameters of the model, which should incorporate any a priori knowl-

edge or assumptions about their values. Given the prior, parameters can be fit by choosing those that maximize the posterior likelihood, which is the product

$$
P(\tau, \sigma, \beta, \boldsymbol{A}) \times P(\text { Data } \mid \tau, \sigma, \beta, \boldsymbol{A})
$$

In the remainder of this section a brief account of the factor $P$ (Data $\mid \tau, \sigma, \beta, \boldsymbol{A}$ ) is given and the assumptions incorporated into the priors are explained.

\section{Likelihood of the data given parameters}

Data from a typical observer consists of several threshold measurements recorded at each of a range of frequencies: the data is denoted as a list of pairs $\left(C_{j, k}, \nu_{j}\right)$ where $\nu_{j}$ ranges over the flicker frequencies at which there are data and, for each fixed value of $j$ (that is, for each fixed flicker frequency), the index $k=1,2, \ldots$ runs over all the available threshold measurements. Given a set of parameters $(\tau, \sigma, \beta, \boldsymbol{A})$ the probability of a observing particular set of data is

$$
P(\text { Data } \mid \tau, \sigma, \beta, \boldsymbol{A})=\prod_{j, k}\left(1-P\left(C_{j, k}, \nu_{j}: \tau, \sigma, \beta, \boldsymbol{A}\right)\right) P\left(\gamma C_{j, k}, \nu_{j}: \tau, \sigma, \beta, \boldsymbol{A}\right) .
$$


This expression, when combined with prior distributions sketched below, enables us to fit the parameters by maximizing the posterior likelihood (2). $\mathrm{C}++$ programs was written to do this, using version 3.1.1 of the discrete Fourier transform library FFTW [23] to compute convolutions (A4) and version 2.4 of Sandia's OPT ++ library [24] to do the optimization.

\section{The parameter $\beta$}

Initial numerical investigations showed that for the data the conditional likelihood (3) de-

pends only weakly on the parameter $\beta$. This makes sense in that $\beta$ controls the slope of the psychometric function, but does not influence the threshold [21]. As only thresholds were measured, the data provide only weak constraints on $\beta$ (though $\beta$ does allow one to predict the variance for repeated threshold measurements at the same frequency). Metha and Mullen [9] addressed a similar issue by measuring the slope of the psychometric function directly, but such measurements were not available and so assumed fixed values of $\beta$ were: 1.73 for the blue stimuli and 2.4 for yellow ones.

\section{Priors on parameters of the filters}

The shapes of Koenderink's family of kernels are characterized by only two parameters, $\tau$ and $\sigma$ [see Eqn. (A3)], but a natural quantitative description of the three filters that are of interest here would require at least five: a roll-off frequency for the low-pass filter and two parameters apiece for each of the band-pass filters; for example, a frequency at peak gain and

a full width at half-maximum. Thus Koenderink's formulation imposes certain inescapable interdependencies among the three filter. To incorporate the expectations about the filters 
a prior distribution on $\tau$ and $\sigma$ proportional to a product of normal distributions designed to constrain features of the gain curves was therefore applied.

The prior, which was the same far all classes of models, whichever combination of filters they included, was

$$
P(\tau, \sigma, \beta, \boldsymbol{A})=P(\tau, \sigma)=\prod_{j=0}^{2} \frac{e^{-\left(f_{j}(\tau, \sigma)-m_{j}\right)^{2} /\left(2 s_{j}^{2}\right)}}{\sqrt{2 \pi s_{j}^{2}}}
$$

where the $f_{j}(\tau, \sigma)$ are frequencies at which certain features of the filters occur, the $m_{j}$ are target values for those frequencies and the $s_{j}$ are tolerances indicating how strictly the targets should be enforced. Thus, for example, $f_{0}(\tau, \sigma)$ is the frequency at which the low-pass filter rolls off to half-maximal gain and $m_{0}=8 \mathrm{~Hz}$ while $s_{0}=3 \mathrm{~Hz}$. This component of the prior acts as a penalty function designed to provide a (fairly weak) constraint that requires the low-pass filter to roll off at $f_{0} \approx 8 \pm 3 H z$. The other two factors in (4) are designed to constrain $f_{1}(\tau, \sigma)$ and $f_{2}(\tau, \sigma)$, which are the frequencies at which the two band-pass filters produce their peak gains. The details of these Gaussian priors are summarized in Table 1. Finally, uniform priors on the filters gains $A_{j}$ were imposed, restricting each to the interval $0 \leq A_{j} \leq 25000$. Numerical experiments show that this range includes all remotely plausible (in terms of fitting the data) values. 


\section{Results}

\section{A. The $1 \mathrm{~Hz}$ spectral sensitivity function}

Figure 1 shows the standard spectral sensitivity function of the cone-opponent mechanisms for two observers on the white background $(3350 \mathrm{~K})$, marked by empty circles. This curve has three characteristic, almost equal, peaks at about 450, 525 and $600-620 \mathrm{~nm}$. A notch at $574 \mathrm{~nm}$ separates the peaks in the red-green spectral range (cf. Ref. 20,25,26) and conceals a response of the yellow-blue mechanism (cf. Ref. 13). The notch is defined as the difference in sensitivity at $574 \mathrm{~nm}$ as compared with at $554 \mathrm{~nm}$ and $601 \mathrm{~nm}$. The notch becomes shallower when luminance intrusions are stronger (cf. Ref. 27). Fig. 1 illustrate this point: spectral sensitivity for a 1-deg spectral spot presented on the white background (empty circles) has a deeper notch than on the yellow background (2520 K), marked by filled circles.

\section{The effect of bar-like stimuli}

The spectral sensitivity curve obtained with the wide bar on a white background (Fig. 1:

empty squares) is only marginally different to that obtained with a 1-deg spot (empty circles), especially for medium-long wavelengths. The notch depth (cf. Ref. 27) at $574 \mathrm{~nm}$ is reduced by about $0.1 \log$ units as compared with the spot, whereas the peak around $450 \mathrm{~nm}$ is reduced by about $0.05 \log$ units.

Narrowing the bar to 0.06 deg (Fig. 1: empty triangles) reduced sensitivity at $450 \mathrm{~nm}$ by 0.6 to $1.0 \log$ unit depending on the observer. The short-wavelength peak is reduced by about $0.3 \log$ units compared with the spot. Thus the sensitivity of the short-wavelength 
cone-opponent mechanism seems to deteriorate as a consequence of introducing spatial edges when a white background is used. There is still a difference between cone-opponent and luminance mechanisms in end-spectral regions, even for the narrow bar: Compare empty triangles with the dashed line in Fig. 1; the dashed line represents $25 \mathrm{~Hz}$ homochromatic flicker for a narrow bar on a white background. The $25 \mathrm{~Hz}$ homochromatic flicker function is identical for the yellow background and is therefore not shown here.

\section{The effect of bar-like stimuli and background color}

A yellow background was introduced to desensitize the L- and M-cone mechanisms (Fig. 1, filled circles). Spectral sensitivity curves measured for bars, either wide or narrow, presented on the yellow background are enhanced for the short-wavelength range as compared with the curves obtained for the white background. Similar effects, which have been known for spot stimuli (Fig. 1, circles), is evident for narrow and wide bars as well (compare filled squares and triangles with the corresponding empty symbols in Fig. 1), indicating that the S-cone opponent mechanism is well separated from the L- and M-cone mechanisms. The shallower notch around $574 \mathrm{~nm}$ may imply that the detection of spectral yellow in this case is influenced by luminance contrast, since the test and background differ little in chromaticity. 


\section{B. The temporal sensitivity function}

\section{The effect of bar-like stimuli}

Actual thresholds and predicted sensitivity curves for spots are plotted in Fig. 2 and for wide and narrow bars in Fig. 3, 4, 5. The temporal frequency sensitivity curves obtained with a 2-deg colored spot on a white background generally gives a low-pass function (cf. Ref. 28). This is similar to what is presented here for a 1-deg blue spot on a yellow background [Fig. 2(b)]. A blue wide bar gives rise to a broader low-pass function with a flat region between 3 to $10 \mathrm{~Hz}$ [Figs. 3 (a) and 4 (a)]. The flat region of the function may be an envelope of the sensitivities of two band-pass (transient) filters.

Tables 2-6 summarize the results from the model fit. The tables have the same format: the likelihood scores for a given stimulus are arranged in a block of four rows; one each for the four classes of model that were tested. Each row begins with a symbol describing the model, for example, $M_{02}$ refers to a model based on the low-pass filter $h_{0}$ and the band-pass filter $h_{2}$, followed by three columns relating to the log of the posterior-likelihood for the maximally likely set of parameters. The column headed Prior lists the log of the Bayesian prior (4) evaluated for the parameters of the most likely model while the column headed Fit is the $\log$ of that factor (3) in the posterior likelihood (2) which gives the probability of seeing the observed data: the latter of these two provides a measure of how well the most likely model in the class actually fits the data. The last column of log-likelihood values, headed Total, gives the sum of the contributions from the prior and the fit and it is this quantity, the log 
of the posterior likelihood (2), that is the target of the likelihood maximization.

The final two columns list $p$-values given by a test of the significance of the differences in log-likelihood between the various models. The test depends on an asymptotic result for the distribution of these differences that requires the models being compared to be nested in the sense that one is a special case of the other. Consider, for example, the models $M_{0}$ and $M_{02}$ : the first includes only a single filter, the low-pass filter $h_{0}$, while the second includes both $h_{0}$ and $h_{2}$, the second of the band-pass filters. Thus $M_{0}$ is a special case of $M_{02}$ for which the amplitude $A_{2}=0$. In the vocabulary of the asymptotic result mentioned above, model $M_{0}$ is said to be nested in model $M_{02}$ and a $\chi^{2}$ test can be used (see, for example, Section 8.4.2 of Ref. 29) to check whether the difference in log-likelihood between the two models is significant. This makes it clear that there are two sorts of significance tests that can be done. As $M_{0}$ is nested within each of the more complex models, it is possible to test all three of them against $M_{0}$ : the $p$-values for these tests are tabulated in the columns headed $p_{1}$. The same framework to test differences in likelihood between models $M_{01}$ and $M_{02}$ can not be used as neither is nested within the other, but both of these two can be tested against the model $M_{012}$. The columns headed $p_{2}$ record $p$-values for the difference in log-likelihood between $M_{012}$ and the most likely member of the pair $M_{01}$ and $M_{02}$.

Tables 2 and 3, top two rows, show that in the case of a blue bar on a white background, a model that combines the low-pass filter with the first band-pass filter (model class $M_{01}$ ), or a model that combines all three filters (model class $M_{012}$ ) provides the best fit to the data. The model that excludes the first band-pass filter and combines the low-pass filter with the 
second band-pass filter (model class $M_{02}$ ) provides the least satisfactory fit to the data. For a narrow blue bar the model $M_{012}$ has a slightly greater likelihood than either $M_{01}$ or $M_{02}$, but the difference is not significant.

\section{The effect of bar-like stimuli and background color}

The predicted temporal-sensitivity curves for a blue wide bar on a yellow background [Figs. 3(c), 4(c), and 5(a)] are similar to those for a blue wide bar on a white background [Figs. 3(a) and 4(a)], this is confirmed in the log-likelihood values for each of the model fits (tables 2 and 3). Table 4 show the result for observer AG for a blue spot and wide and narrow blue bars on a yellow background; his results show the same trend. This indicates that a transient-1 filter also subserves detection of S-cone stimuli. Narrowing the bar shifts the peak of the predicted sensitivity curves to lower temporal frequencies, as a wide bar, detected by its two edges, is known to engage faster processing than a narrow bar (cf. Ref. $30)$.

A yellow bar, wide or narrow, on a yellow background gives rise to a temporal sensitivity curve with a peak around $10 \mathrm{~Hz}$ [Figs. 3(e) and (f) and 4(e) and (f)]. Tables 5 and 6 show that the model that fits a yellow spot or bar best is either the model that combines the low-pass filter with the second band-pass filter (model class $M_{02}$ ), or a model that combines

all three filters (model class $M_{012}$ ). The model that combines the low-pass filter with the first band- pass filter (model class $M_{01}$ ) provides the poorest fit to the data. 


\section{Discussion}

The results show that at least two temporal frequency-selective filters (or channels) are necessary to account for detection of both chromatic (blue bars on a yellow or a white background) and achromatic (yellow bars on a yellow background) stimuli. The transient-2 filter dominates the predicted sensitivity curves obtained with the yellow bars, whereas the transient-1 filter is the transient filter that dominates the predicted sensitivity curves obtained with the blue bars. This transition reveals a shift in relative contributions of color and luminance from low to higher temporal frequencies, and we argue that interaction between color and luminance signals drives visual processing at intermediate temporal frequencies.

The deterioration of the cone-opponent function as a consequence of change of shape from a spot to a rectangular bar can be inferred from the change in the spectral sensitivity functions (Fig. 1). One plausible explanation for the observation is that bar-like stimuli (presented at 1 $\mathrm{Hz}$ ) activate a hypothetical intermediate detection mechanism operating between pure color, mediated by the sustained temporal filter, and pure luminance, mediated by the transient-2 temporal filter. This is not an effect of area since the change in effective area of a spot, from $0.1 \mathrm{deg}^{2}$ to above $1 \mathrm{deg}^{2}$, only produces a vertical shift in sensitivity [19]. Note that patterndetection sensitivity is known to increase when straight edges are present in the stimuli [31], unlike pure luminance detection, which is best for large spots [1].

The presence of straight edges in the stimuli still increases the activation of the luminance mechanism as compared with the cone-opponent mechanism, and this is evident in the spec- 
tral sensitivity function as the notch around $575 \mathrm{~nm}$ is shallower and end-spectral sensitivity at 450 and $600 \mathrm{~nm}$ is reduced for the narrow and wide bar stimuli as compared with the spot stimulus (Fig. 1). These results are in line with previous quantitative assessments of contribution of cone-opponent versus luminance mechanism around $575 \mathrm{~nm}$ [13,20,32-37]. Reviewing the experimental evidence available, Sharanjeet-Kaur et al. [27] concluded that circular blurred spots, or spots with an annulus, are optimal stimuli to activate cone-opponent mechanisms. Introduction of a yellow background further increases chance achromatic detection $[13,15]$, and makes it difficult to separate the yellow chromatic part of the blue-yellow cone-opponent mechanism, especially if the wavelengths of the test stimulus and background are similar [13]. Conversely, the yellow background accentuates chromatic detection for a stimulus with peak wavelength around $450 \mathrm{~nm}$ as a consequence of L- and M-cone desensitizing. Luminance contrast is thereby unlikely to be responsible for detection at $450 \mathrm{~nm}$, and thus the results for blue stimuli suggest different processes.

Stimuli with straight edges like the bar-like stimuli employed here are not optimal shapes for stimulation of the cone-opponent mechanisms as spatial edges reduces the peaks at both ends of the visible spectrum as shown previously [38]. Narrowing the bars reduces the spectral sensitivity of the peak around $450 \mathrm{~nm}$ even more, probably because of foveal tritanopia $[39,40]$. Apparently, even though the sensitivity of the cone-opponent mechanism to the bar-like stimuli is lower than for spots, the sensitivity of the luminance mechanism under such viewing conditions is even lower (see Fig. 1 dashed line the $25 \mathrm{~Hz}$ homochromatic flicker function approximates the luminosity function). The luminance mechanism does not 
contribute much in the spectral sensitivity function at both ends of the visible spectrum. Detection of wide blue bars is thus mediated mainly by the cone-opponent mechanism. Furthermore, measurements performed on yellow backgrounds, show an overall increase of sensitivity around $450 \mathrm{~nm}$, both for wide and narrow bars, indicating good separation of the S-cone response.

In spite of the quantitative differences in the spectral sensitivity curves for the various conditions of detection of narrow and wide blue bars, the predicted temporal sensitivity curves reveal the presence of the same intermediate (transient-1) filter for blue bars on white and yellow backgrounds. Moreover, the models that include the transient-1 filter also provides the best fit to the data (e.g. Table 2).

\section{A. The temporal sensitivity function}

The detection of a blue spot on a white background by cone-opponent mechanism is well separated from the luminance mechanism, as defined by homochromatic flicker photometry. The temporal frequency curve under this condition is low-pass with a fall-off at $10 \mathrm{~Hz}[28$, 41]. The predicted sensitivity curves obtained for a blue bar with both white and yellow background conditions are flat between 3 and $10 \mathrm{~Hz}$ with lower sensitivity at low temporal frequencies [Figs. 3 and 4 (a) and (b)] than for a 1-deg spot stimulus [compare Fig. 2 (b) with Figs. 3 (c), 4 (c) and 5 (a)]. The flattening at intermediate frequencies of the temporal sensitivity curves reflects that under conditions were spatial edges are prominent, the coneopponent mechanism, which is believed to be of sustained properties only, is not operating 
on its own. The argument put forward here is that this is because perception is mediated by the transient-1 filter. The model employed to derive the theoretical filters underlying the data support that at least two filters are necessary to account for the data, with models encompassing both the sustained and the transient-1 filters being favoured. A similar effect was demonstrated with different intensity levels of the yellow background [42], giving a flatter region between 4 and $9 \mathrm{~Hz}$ at lower levels of background illumination. Stockman et al. also reported that S-cone signals are mediated by two temporal filters [43]; the sustained and the transient-2 filters. They used high intensity levels that shift sensitivity to higher temporal frequencies; this may be why they did not consider the transient-1 filter. Any contribution of the transient-1 filter has been shown to be evident if the temporal frequency sensitivity curve exhibits a peak around $4 \mathrm{~Hz}[6,44]$. Involvement of a distinctive intermediate transient component has been reported for red-green isoluminant stimuli and has been attributed to the operation of an additional chromatic mechanism $[10,45]$. It has been demonstrated by two other separate investigations $[46,47]$ that under strict conditions of isoluminance, and after accounting for this transient component, that both red-green and blue-yellow (tritan) cone-opponent mechanisms are purely low-pass at threshold, referred to as pure color. This implies that the cone-opponent mechanism cannot be the sole operator over the range of intermediate temporal frequencies. 


\section{Related studies}

The three temporal frequency-selective filters apparent in the data presented here are most likely to represent the following: a sustained filter subserving perception of cone-opponent processing without luminance intrusion (also referred to as pure color), one transient-1 filter of mixed color and luminance properties, and a transient-2 filter of pure luminance properties. While retrospectively analyzing early studies it became apparent that the intermediate transient-1 filter was actually evident in the data of Robson [48] (counter-phased luminance gratings) and Kelly [8] (a bipartite field). Kulikowski and Tolhurst [6] revealed two different temporal frequency-selective filters for oriented achromatic stimuli. Furthermore, two discrete temporal frequency-selective filters were reported for detecting chromatic and oriented stimuli [42] and straight-line contours [49]. Finally, a similar finding has been reported in S-cone monochromatic observers [50].

The slow low-pass (sustained) filter was identified as exclusively serving fine pattern detection above $25 \mathrm{c} / \mathrm{deg}$ [51]. A similar filter is also serving the detection of color as reviewed recently $[46,47]$. On the other hand, the transient-1 filter, responsible for motion perception, is stronger for spatial patterns, and is clearly separable from pure flicker detection [1]. The so-called movement mechanism, optimal around 3-7 Hz and $2 \mathrm{c} / \mathrm{deg}$, depending on level of retinal illumination, may not have any contribution from the transient-2 filter (pure flicker mechanism). Burr and Ross [44] have shown that most spatio-temporal contributions to the detection of fast motion are due to a transient filter with peak sensitivity around $4 \mathrm{~Hz}$ (the 
transient-1 filter). The predominance of the transient-2 filter over the transient-1 filter is shown only above $25 \mathrm{~Hz}$ for flashed spots [41].

\section{Chromatic-pattern mechanism: possible neuronal basis}

S-, M- and L-cones make characteristic connections in the retina via bipolar neurons (for review see: [52]). S-cones do not project to magnocellular ganglion neurons. Dacey and Lee [53] have shown that excitatory S-cone input goes via specific bipolar neurons to distinct bistratified ganglion neurons and the koniocellular pathway [54] there from. S-cone inhibitory connections are associated with different neurons $[55,56]$. Most numerous are L-M opponent connections forming the parvocellular pathway $[57,58]$. In spite of these morphological differences, the $+\mathrm{S}-(\mathrm{L}),+\mathrm{S}-(\mathrm{M})$ and L-M opponent ganglion and LGN neurons have temporal characteristics that are not substantially different [56], and all exceed psychophysical limits of temporal resolution [59]. It is argued that the perceptual limits of temporal resolution must be determined at the cortical level [60]. Magnocellular neurons receive information as a non-linear sum from L+M cones; their spectral characteristics fit the V-lambda luminosity function best and are often regarded as substrate of luminance information [61-63]; but see [64] for an alternative view. Although it was argued that neurons that receive parvocellular input responds in a way that can approximate the V-lambda luminosity function [65], both parvo- and koniocellular neurons have been reported to have too low luminance contrast sensitivity $[56,66-68]$ to contribute on their own to luminance contrast detection. Studies of single neurons in the monkey primary cortex V1, have revealed distinct color neurons 
that were not sensitive to luminance contrast [69-71], but in addition also many neurons that were sensitive to both color and luminance contrast. The latter are typically neurons with elongated receptive fields [72-78]. Mixing of parvo-, konio- and magnocellular pathways have been demonstrated anatomically in area V1 $[68,79,80]$. Moreover, neruons that received mixed input from the three pre-cortical pathways had the highest maximum achromatic contrast sensitivity at low spatial frequencies and moderate velocities. These composite neurons may form the basis of a mechanism that combines information of color, form and motion.

Kulikowski and Walsh $[81,82]$ introduced a concept of a chromatic-pattern, motion and stereo detection mechanism, which may process a mixture of color and luminance signals, possibly reflecting an integration of konio-, parvo- and magnocellular inputs. Its spectral characteristic depends critically on the shape of the stimulus; for a 1-deg spot it is the envelope of typical cone-opponent functions [20,83]; for rectangular stimuli, it is a weighted characteristic of the V-lambda luminosity function and the two cone-opponent mechanisms: red-green and blue-yellow.

This view is consistent with the finding of Metha and Mullen [10], who examined redgreen isoluminant and luminance modulated Gabor patches involved in temporal frequency discrimination. They concluded that at least two temporal filters must be involved which correspond to the sustained and the transient-1 filter. They suggested that the transient-1 filter might be part of the color detection process. However, McKeefry [46] argued that when the transient-1 filter is excluded in an aberration-free experiment, red-green and blue-yellow sensitivity become equal and color is processed in a veridical manner. This would suggest 
separate systems for color and chromatic pattern analyzis, the latter would depend on the shape of the visual stimuli.

\section{Conclusion}

The data presented here demonstrate the involvement of a transient-1 filter in a task requiring the detection of bar-like stimuli with combined color and luminance contrast. Detection of spectral spots is predominantly executed by the cone-opponent mechanism (a sustained filter) at low temporal frequencies, and by the pure flicker (luminance) mechanism (the transient-2 filter) at very high temporal frequencies. The involvement of the transient-1 filter for detecting bar-like stimuli suggests that this temporal frequency-selective filter is linked with analyzis of chromatic patterns, integrating color and form in to a unified percept (cf. Ref. 84). It is postulated that this intermediate mechanism which is based on mixed konio-, parvo- and magnocellular input to some orientation sensitive visual cortical units [68] affect perception. The change of stimulus shape may critically alter the involvement of these units. 


\section{A. Probability summation model for the method of adjustment: mathematical}

\section{details}

Watson's probability summation model [21] requires two ingredients: a set of filters and a probabilistic detection model. For the first, we followed Metha and Mullen [9] and built models based on combinations of three filters - a low-pass filter and two band-pass filterswhose impulse response functions are

$$
\begin{aligned}
h_{0}(t: \tau, \sigma) & =\exp \left\{-\frac{(\ln (t / \tau))^{2}}{\sigma^{2}}\right\} \\
h_{1}(t: \tau, \sigma) & =d h_{0} / d t \\
& =-\left(\frac{2}{t \sigma^{2}}\right) \ln (t / \tau) \exp \left\{-\frac{(\ln (t / \tau))^{2}}{\sigma^{2}}\right\} \\
& =-\left(\frac{2}{t \sigma^{2}}\right) \ln (t / \tau) h_{0}(t) \\
h_{2}(t: \tau, \sigma) & =d h_{1} / d t \\
& =-\left(\frac{2}{t^{2} \sigma^{4}}\right)\left[\frac{2}{\sigma^{4}} \ln (t / \tau)^{2}+\ln (t / \tau)-1\right] \exp \left\{-\frac{(\ln (t / \tau))^{2}}{\sigma^{2}}\right\} \\
& =-\left(\frac{2}{t^{2} \sigma^{2}}\right)\left[\frac{2}{\sigma^{2}}(\ln (t / \tau))^{2}+\ln (t / \tau)-1\right] h_{0}(t) .
\end{aligned}
$$

Here the parameters $\tau$ and $\sigma$ characterize, respectively, the rise-times and temporal durations of responses.

If the stimulus flickers periodically with contrast $C$, frequency $\nu$ (in Hz.) and temporal waveform $g(t: \nu)$ then the responses of the various filters are given by convolutions:

$$
\begin{aligned}
R_{j}(t) & =C g(t: \nu) * h_{j}(t: \tau, \sigma) \\
& =C \int_{0}^{\infty} g(t-s: \nu) h_{j}(s: \tau, \sigma) d s
\end{aligned}
$$


where, on the left-hand side, the dependence on the parameters $\tau$ and $\sigma$ has been suppressed.

Note that as the waveforms $g(t: \nu)$ are periodic with period $1 / \nu$, so are the responses $R_{j}(t)$.

In terms of the $R_{j}(t)$, the probability that a subject would fail to detect the stimulus during some interval $T_{0} \leq t \leq T_{1}$ is given by

$$
P_{W}(C, \nu: \tau, \sigma, \beta, \boldsymbol{A})=2^{-\int_{T_{0}}^{T_{1}} \sum_{j=0}^{2}\left|A_{j} R_{j}(t)\right|^{\beta} d t}
$$

where here the dependence on all parameters is shown explicitly, including the vector of amplitudes $\boldsymbol{A}=\left(A_{0}, A_{1}, A_{2}\right)$ and the Weibull parameter $\beta$ that comes from Watson's probabilistic detection model.

Application of (A5) is straightforward when the signal is presented for a finite period (thus fixing the bounds $T_{0}$ and $T_{1}$ of the integral), but has no immediately obvious interpretation when, as in our experiments, the flickering stimulus is presented essentially continuously, with the subject deciding whether the stimulus is visible or not and choosing when to modify the contrast $C$. In this context there are two natural generalizations to Eqn. (A5), both of which rely on some implicit "effective presentation interval" during which the subject attends to the stimulus to decide whether it is visible or not.

In the first formulation, which we will call the constant $T$ formulation, the subject is assumed to study the stimulus for a fixed time $T$, independent of the flicker frequency. In the second formulation, the constant $N$ formulation, we assume that the subject observes the stimulus for a fixed number of cycles, $N$, of the flickering. In both formulations we assume that after the observer adjusts the contrast he or she then ignores the stimulus for a 
sufficiently long time that transients may be neglected and we can be assume that $R_{j}(t)$ is periodic.

But as the responses are assumed periodic there is, in both formulations, an issue about the point in the cycle of the periodic flickering at which the observer begins to attend to the stimulus. That is, there is some unknown phase $0 \leq \theta \leq 1$ such that the limits of the integral in (A5) become

$$
T_{0}=\theta / \nu \quad \text { and } \quad T_{1}= \begin{cases}(\theta / \nu)+T & \text { In the constant } T \text { formulation } \\ (\theta+N) / \nu & \text { In the constant } N \text { formulation }\end{cases}
$$

And as there is no way of knowing $\theta$, one must average over all possible values, replacing the integral in the exponent of (A5) with, for example in the constant $N$ formulation,

$$
\begin{aligned}
\int_{0}^{1} d \theta \int_{\theta / \nu}^{(\theta+N) / \nu} \sum_{j=0}^{2}\left|A_{j} R_{j}(t)\right|^{\beta} d t & =\int_{0}^{N / \nu} \sum_{j=0}^{2}\left|A_{j} R_{j}(t)\right|^{\beta} d t \\
& =N\left[\int_{0}^{1 / \nu} \sum_{j=0}^{2}\left|A_{j} R_{j}(t)\right|^{\beta} d t\right]
\end{aligned}
$$

where, in simplifying the expression, we have used the fact that the integral of a periodic function over $N$ complete periods is independent of starting time and equal to $N$ times the integral over a single period. Notice that the final line is proportional to $N$, but that no $N$ 's appear inside the square brackets surrounding the integral: integrals over a single period of the responses $R_{j}(t)$ are all that need be computed.

In the constant $T$ formulation the corresponding calculation is

$$
\int_{0}^{1} d \theta \int_{\theta / \nu}^{(\theta / \nu)+T} \sum_{j=0}^{2}\left|A_{j} R_{j}(t)\right|^{\beta} d t=\int_{0}^{1} d \theta \int_{0}^{T} \sum_{j=0}^{2}\left|A_{j} R_{j}(s+\theta / \nu)\right|^{\beta} d s
$$


where, in passing from the left hand side to the right, we have changed the variable of integration to $s=t-\theta / \nu$. Then, interchanging the order of integration, we obtain the following

$$
\int_{0}^{1} d \theta \int_{0}^{T} \sum_{j=0}^{2}\left|A_{j} R_{j}(s+\theta / \nu)\right|^{\beta} d s=\int_{0}^{T} d s \int_{0}^{1} \sum_{j=0}^{2}\left|A_{j} R_{j}(s+\theta / \nu)\right|^{\beta} d \theta
$$

To continue with this calculation it is convenient to make a second change of variable, defining $\eta=\theta / \nu:$

$$
\begin{aligned}
\int_{0}^{T} d s \int_{0}^{1} \sum_{j=0}^{2}\left|A_{j} R_{j}(s+\theta / \nu)\right|^{\beta} d \theta & =\int_{0}^{T} d s \int_{0}^{1 / \nu} \sum_{j=0}^{2}\left|A_{j} R_{j}(s+\eta)\right|^{\beta} \nu d \eta \\
& =\int_{0}^{T} \nu d s \int_{0}^{1 / \nu} \sum_{j=0}^{2}\left|A_{j} R_{j}(s+\eta)\right|^{\beta} d \eta
\end{aligned}
$$

Finally, again using the fact that the integral of a periodic function over a complete period is independent of the starting point, we obtain:

$$
\begin{aligned}
\int_{0}^{T} \nu d s \int_{0}^{1 / \nu} \sum_{j=0}^{2}\left|A_{j} R_{j}(s+\eta)\right|^{\beta} d \eta & =\int_{0}^{T} \nu d s\left[\int_{0}^{1 / \nu} \sum_{j=0}^{2}\left|A_{j} R_{j}(\eta)\right|^{\beta} d \eta\right] \\
& =\nu T\left[\int_{0}^{1 / \nu} \sum_{j=0}^{2}\left|A_{j} R_{j}(\eta)\right|^{\beta} d \eta\right]
\end{aligned}
$$

This result has a certain formal similarity to (A6): both results are proportional to the integral of $\left|R_{j}(t)\right|^{\beta}$ over a single period, but the fixed- $T$ result includes a factor of the frequency $\nu$ that is absent from Eqn. (A6).

Armed with these results, it is easy to write down a probability of non-detection:

$$
P(C, \nu: \tau, \sigma, \beta, \boldsymbol{A})= \begin{cases}2^{-\nu T\left[\int_{0}^{1 / \nu} \sum_{j=0}^{2}\left|A_{j} R_{j}(\eta)\right|^{\beta} d \eta\right]} & \text { Constant } T \text { formulation } \\ 2^{-N\left[\int_{0}^{1 / \nu} \sum_{j=0}^{2}\left|A_{j} R_{j}(\eta)\right|^{\beta} d \eta\right]} & \text { Constant } N \text { formulation }\end{cases}
$$


But these formulae are meant to be used as the basis for the maximum-likelihood fitting program outlined in Section A and, for this, they include too many parameters. To see why, consider the expression for the exponent in the constant $N$ formulation:

$$
\begin{aligned}
N\left[\int_{0}^{1 / \nu} \sum_{j=0}^{2}\left|A_{j} R_{j}(\eta)\right|^{\beta} d \eta\right] & =\sum_{j=0}^{2} N\left[\int_{0}^{1 / \nu}\left|A_{j} R_{j}(\eta)\right|^{\beta} d \eta\right] \\
& =\sum_{j=0}^{2} N A_{j}^{\beta}\left[\int_{0}^{1 / \nu}\left|R_{j}(\eta)\right|^{\beta} d \eta\right] .
\end{aligned}
$$

The last line emphasizes the fact that the probability of non-detection depends only on the products $N A_{j}{ }^{\beta}$, which means that if one were to change $N$ to, say, $N^{\prime}=\alpha N$ and, at the same time, change all the filter amplitudes to $A_{j}^{\prime}=A_{j} / \alpha^{1 / \beta}$ then the products that enter into (A8) would remain unchanged:

$$
N^{\prime}\left(A_{j}^{\prime}\right)^{\beta}=(\alpha N)\left(\frac{A_{j}}{\alpha^{1 / \beta}}\right)^{\beta}=(\alpha N)\left(\frac{A_{j}{ }^{\beta}}{\alpha}\right)=N A_{j}{ }^{\beta}
$$

In other words, one cannot hope to find a unique, best-fitting set of parameters if one permits both $N$ and the $A_{j}$ to vary. Alternatively one could say that the expressions (A9) are unsuited to our data in the sense that, without further measurement, we cannot distinguish between a very deliberate observer who looks at the signal for a long time (has a large $N$ or $T$ ), but has rather small filter amplitudes $A_{j}$, and a quicker observer who looks briefly (i.e., has $N^{\prime}<<N$ or $\left.T^{\prime}<<T\right)$, but compensates with larger filter amplitudes $A_{j}^{\prime}$.

To get rid of this ambiguity we fix one of the parameters, choosing $T=1$ in the constant $T$ formulation and $N=1$ in the constant $N$ formulation. This leads the following expressions, 
which yield a well-posed, maximum-likelihood parameter-fitting problem:

$$
P(C, \nu: \tau, \sigma, \beta, \boldsymbol{A})= \begin{cases}2^{-\nu\left[\int_{0}^{1 / \nu} \sum_{j=0}^{2}\left|A_{j} R_{j}(\eta)\right|^{\beta} d \eta\right]} & \text { Constant } T \text { formulation } \\ 2^{-\left[\int_{0}^{1 / \nu} \sum_{j=0}^{2}\left|A_{j} R_{j}(\eta)\right|^{\beta} d \eta\right]} & \text { Constant } N \text { formulation }\end{cases}
$$

These probabilities are the main ingredient required for the analysis in Section A.

\section{Acknowledgments}

The authors thank D. Carden and J. Simpson for their technical assistance; A.G. Robson for serving as observer; J. S. Werner, K. T. Mullen, K. Kranda and D. H. Foster for comments on

earlier drafts; R. C. Baraas is funded by the Research Council of Norway Grant 182768/V10. 


\section{References}

1. P. E. King-Smith and J. J. Kulikowski, "Pattern and flicker detection analysed by subthreshold summation," Journal of Physiology 249, 519-548 (1975).

2. M. B. Mandler and W. Makous, "A three channel model of temporal frequency perception," Vision Research 24, 1881-1887 (1984).

3. S. J. Anderson and D. C. Burr, "Spatial and temporal selectivity of the human motion detection system," Vision Research 25, 1147-1154 (1985).

4. R. F. Hess and G. T. Plant, "Temporal frequency discrimination in human vision: Evidence for an additional mechanism in the low spatial and high temporal frequency region," Vision Research 25, 1493-1500 (1985).

5. R. F. Hess and R. J. Snowden, "Temporal properties of human visual filters: number, shapes and spatial covariation," Vision Research 32, 47-59 (1992).

6. J. J. Kulikowski and D. J. Tolhurst, "Psychophysical evidence for sustained and transient detectors in human vision," Journal of Physiology 232, 149-162 (1973).

7. J. J. Kulikowski, "Some stimulus parameters affecting spatial and temporal resolution of human vision," Vision Research 11, 83-93 (1971).

8. D. H. Kelly, "Luminous and chromatic flickering patterns have opposite effects," Science 188, 371-372 (1975).

9. A. B. Metha and K. T. Mullen, "Temporal mechanisms underlying flicker detection and identification for red-green and achromatic stimuli," Journal of the Optical Society of 
America A 13, 1969-1980 (1996).

10. A. B. Metha and K. T. Mullen, "Red-green and achromatic temporal filters: a ratio model predicts contrast-dependent speed perception," Journal of the Optical Society of America A 14, 984-996 (1997).

11. C. F. Stromeyer. III, R. E. Kronauer, A. Ryu, A. Chaparro, and R. T. Eskew. Jr., "Contributions of human long-wave and middle-wave cones to motion detection," Journal of Physiology 485, 221-243 (1995).

12. C. F. Stromeyer. III, A. Chaparro, A. S. Tolias, and R. E. Kronauer, "Colour adaptation modifies the long-wave versus middle-wave cone weights and temporal phases in human luminance (but not red-green) mechanism," Journal of Physiology 449, 227-254 (1997).

13. Sharanjeet-Kaur, J. J. Kulikowski, and V. Walsh, "The detection and discrimination of categorical yellow," Ophthalmic and Physiological Optics 17, 32-37 (1997).

14. M. Marré, "The investigation of acquired colour vision deficiencies," in Colour, pp. 99135 (Adam Hilger, London, 1973).

15. A. Nacer, I. J. Murray, and J. J. Kulikowski, "Balancing sensitivity of human chromatic opponent mechanisms by adaptation," Journal of Physiology P21, 485P (1995).

16. K. T. Mullen and J. J. Kulikowski, "Wavelength discrimination at detection threshold," Journal of the Optical Society of America A 7, 733-742 (1990).

17. G. Westheimer, "The Maxwellian view," Vision Research 6, 669-682 (1966).

18. F. W. Campbell and J. J. Kulikowski, "Orientational selectivity of the human visual 
system," Journal of Physiology 187, 437-445 (1966).

19. A. Nacer, "The interaction between chromatic and achromatic mechanisms of human colour vision: Limitations of sensitivity," Ph.D. thesis, Department of Optometry and Vision Sciences, UMIST, Manchester, UK (1990).

20. P. E. King-Smith and D. Carden, "Luminance and opponent-colour contributions to visual detection and adaptation and to temporal and spatial integration," Journal of the Optical Society of America 66, 709-717 (1976).

21. A. B. Watson, "Probability summation over time," Vision Research 19, 515-522 (1979).

22. J. J. Koenderink, "Scale-Time," Biological Cybernetics 58, 159-162 (1988).

23. M. Frigo and S. G. Johnson, "The Design and Implementation of FFTW3," Proceedings of the IEEE 93(2), 216-231 (2005). URL http://www.fftw.org/.

24. J. Meza, R. Oliva, P. Hough, and P. Williams, "OPT++: An Object Oriented Toolkit for Nonlinear Optimization," ACM Transactions on Mathematical Software 33(2), Article No. 12 (2007). URL http://doi.acm.org/10.1145/1236463.1236467.

25. H. G. Sperling and R. S. Harwerth, "Red-green cone interactions in the increment threshold spectral sensitivity of primates," Science 172, 180-184 (1971).

26. D. H. Foster and R. S. Snelgar, "Test and field spectral sensitivities of colour mechanisms obtained on small white backgrounds: action of unitary opponent-colour processes?," Vision Research 23, 787-797 (1983).

27. Sharanjeet-Kaur, J. J. Kulikowski, and D. Carden, "Isolation of chromatic and achro- 
matic mechanisms: A new approach," Ophthalmic and Physiological Optics 18, 49-56 (1998).

28. H. D. Lange Dzn, "Research into the dynamic nature of the human fovea-cortex systems with intermittent and modulated light. I. Attenuation characteristics with white and colored light," Journal of the Optical Society of America 48, 777-784 (1958).

29. W. J. Ewens and G. R. Grant, Statistical Methods in Bioinformatics: An Introduction (Springer Verlag, New York, 2001).

30. P. E. King-Smith and J. J. Kulikowski, "Line, edge and grating detectors in human vision," Journal of Physiology 230, 23P-25P (1973).

31. J. Bacon and P. E. King-Smith, "The detection of line segments," Perception 6, 125-131 (1977).

32. C. R. I. Jr. and B. A. Drum, "How neural adaptation changes chromaticity coordinates," Journal of the Optical Society of America 63(3), 369-373 (1973).

33. C. R. Ingling. Jr., E. Martinez, and A. L. Lewis, "Tonic-phasic-channel dichotomy and Crozier's law," Journal of the Optical Society of America 73, 183-189 (1983).

34. J. J. Kulikowski and K. Kranda, "Detection of coarse patterns with minimum contribution from rods," Vision Research 17, 653-656 (1977).

35. J. E. Thornton and E. N. Pugh, Jr., "Red/green colour opponency at detection threshold," Science 219, 191-193 (1983).

36. R. S. Snelgar, D. H. Foster, and M. O. Scase, "Isolation of opponent-colour mechanisms 
at increment threshold," Vision Research 27, 1017-1027 (1987).

37. D. J. Calkins, J. E. Thornton, and E. N. Pugh, Jr., "Monochromatism determined at a long-wavelength/middle-wavelength cone-antagonistic locus," Vision Research 32, 23492367 (1992).

38. R. C. Baraas, J. J. Kulikowski, and A. R. Robson, "Spatial edges reduce colour selectivity," Perception 27S, 168-169 (1998).

39. D. R. Williams, D. I. MacLeod, and M. M. Hayhoe, "Punctate sensitivity of the bluesensitive mechanism," Vision Research 21, 1357-1375 (1981).

40. D. R. Williams, D. I. A. MacLeod, and M. M. Hayhoe, "Foveal tritanopia," Vision Research 21, 1341-1356 (1981).

41. A. Nacer, I. J. Murray, Sharanjeet-Kaur, and J. J. Kulikowski, "Selectivity limits of spectral sensitivity functions for chromatic and achromatic mechanisms," in John Dalton's Colour Vision Legacy, pp. 83-91 (Taylor \& Francis Ltd., London, 1997).

42. J. J. Wisowaty and R. M. Boynton, "Temporal modulation sensitivity of the blue mechanism: measurements made without chromatic adaptation," Vision Research 20, 895-909 (1980).

43. A. Stockman, D. I. A. MacLeod, and D. D. DePriest, "The temporal properties of the human short-wave photoreceptors and their associated pathways," Vision Research 31, 189-208 (1991).

44. D. C. Burr and J. Ross, "Contrast sensitivity at high velocities," Vision Research 22, 
479-484 (1982).

45. D. J. McKeefry and J. J. Kulikowski, "Spatial and temporal sensitivities of colour discrimination mechanisms," in John Dalton's Colour Vision Legacy, pp. 163-172 (Taylor \& Francis Ltd., London, 1997).

46. D. M. McKeefry, I. J. Murray, and J. J. Kulikowski, "Red-green and blue-yellow mechanisms are matched in sensitivity for temporal and spatial modulation," Vision Research 41, 245-255 (2001).

47. A. G. Shapiro, L. A. Baldwin, and J. D. Mollon, "The S and L-M chromatic system have matched temporal processing characteristics only at low-light levels," Perception 31, S68b (2002).

48. J. G. Robson, "Spatial and temporal contrast sensitivity of the human eye," Journal of the Optical Society of America 56, 1141-1150 (1966).

49. P. Cavanagh, D. I. A. MacLeod, and S. M. Anstis, "Equiluminance: spatial and temporal factors and the contribution of blue-sensitive cones," Journal of the Optical Society of America A 4, 1428-1438 (1987).

50. R. F. Hess, K. T. Mullen, and E. Zrenner, "Human photopic vision with only short wavelength cones: post-receptoral properties," Journal of Physiology 417, 151-172 (1989).

51. M. H. A. Russell, J. J. Kulikowski, and I. J. Murray, "Spatial frequency dependence of the human visual evoked potential," in Evoked Potentials III, pp. 231-239 (Butterworth, Baltimore, 1987). 
52. P. R. Martin, "Colour processing in the primate retina: recent progress," Journal of Physiology 513, 631-638 (1998).

53. D. M. Dacey and B. B. Lee, "The 'blue-on' opponent pathway in primate retina originates from a distinct bistratified ganglion cell type," Nature 367, 731-735 (1994).

54. P. R. Martin, A. J. R. White, A. K. Goodchild, H. D. Wilder, and A. E. Sefton, "Evidence that blue-on cells are part of the third geniculocortical pathway in primates," European Journal of Neuroscience 9, 1536-1541 (1997).

55. D. J. Calkins, "Representation of cone signals in the primate retina," Journal of the Optical Society of America A 17, 597-606 (2000).

56. C. Tailby, S. G. Solomon, and P. Lennie, "Functional asymmetries in visual pathways carying S-cone signals in Macaque," Journal of Neuroscience 28, 4078-4087 (2008).

57. M. S. Livingstone and D. H. Hubel, "Anatomy and physiology of a color system in the primate visual cortex," Journal of Neuroscience 4, 309-356 (1984).

58. D. Y. Ts'o and C. D. Gilbert, "The organization of chromatic and spatial interactions in the primate striate cortex," Journal of Neuroscience 8, 1712-1728 (1988).

59. T. Yeh, B. B. Lee, and J. Kremers, "Temporal response of ganglion cells of the macaque retina to cone-specific modulation," Journal of the Optical Society of America A 12, 456-464 (1995).

60. J. Cass, C. W. G. Clifford, D. Alais, and B. Spehar, "Temporal structure of chromatic channels revealed through masking," Journal of Vision 9(5)(17), 1-15 (2009). 
61. B. B. Lee, P. R. Martin, and A. Valberg, "Nonlinear summation of M- and L-cone inputs to phasic retinal ganglion cells of the macaque," Journal of Neuroscience 9, 1433-1442 (1989).

62. B. B. Lee, J. Pokorny, V. C. Smith, P. R. Martin, and A. Valberg, "Luminance and chromatic modulation sensitivity of macaque ganglion cells and human observers," Journal of the Optical Society of America A 7, 2223-2236 (1990).

63. P. Lennie, J. Pokorny, and V. C. Smith, "Luminance," Journal of the Optical Society of America A 10, 1283-1293 (1993).

64. W. H. Merigan and J. H. Maunsell, "Macaque vision after magnocellular lateral geniculate lesions," Visual Neuroscience 5, 347-352 (1990).

65. P. Gouras and E. Zrenner, "Enchancement of luminance flicker by color-opponent mechanisms," Science 205, 587-589 (1979).

66. E. Kaplan and R. M. Shapley, "X and Y cells in the lateral geniculate nucleus of macaque monkeys," Journal of Physiology 330, 125-143 (1982).

67. T. P. Hicks, B. B. Lee, and T. R. Vidyasagar, "The responses of cells in macaque lateral geniculate nucleus to sinusoidal gratings," Journal of Physiology 337, 183-200 (1983).

68. T. R. Vidyasagar, J. J. Kulikowski, D. M. Lipnicki, and B. Dreher, "Convergence of parvocellular and magnocellular information channels in the primary visual cortex of the macaque," European Journal of Neuroscience 16(5), 945-956 (2002).

69. T. Yoshioka and B. M. Dow, "Color, orientation and cytochrome oxidase reactivity in 
areas V1, V2 and V4 of macaque monkey visual cortex," Behavioural Brain Research 76, 71-88 (1996).

70. T. Yoshioka, B. M. Dow, and R. G. Vautin, "Neuronal mechanisms of color categorization in areas V1, V2 and V4 of macaque monkey visual cortex," Behavioural Brain Research 76, 51-70 (1996).

71. J. J. Kulikowski and V. Walsh, "Colour vision: isolating mechanisms in overlapping streams," Progress in Brain Research 95, 417-426 (1993).

72. C. R. Michael, "Color-sensitive complex cells in monkey striate cortex," Journal of Neurophysiology 41, 1250-1266 (1978).

73. C. R. Michael, "Color vision mechanisms in monkey striate cortex: simple cells with dual opponent-color receptive fields," Journal of Neurophysiology 41, 1233-1249 (1978).

74. R. G. Vautin and B. M. Dow, "Color cell groups in foveal striate cortex of the behaving macaque," Journal of Neurophysiology 54, 273-292 (1985).

75. R. L. De Valois and K. K. De Valois, "Neural coding of color," in Handbook of Perception, vol. 5, pp. 117-166 (Academic Press, New York, 1975).

76. R. L. De Valois and K. K. De Valois, "A multi-stage color model," Vision Research 33, 1053-1065 (1993).

77. P. Lennie, J. Krauskopf, and G. Sclar, "Chromatic mechanisms in striate cortex of macaque," Journal of Neuroscience 10, 649-669 (1990).

78. E. N. Johnson, M. J. Hawken, and R. Shapley, "The spatial transformation of color 
in the primary visual cortex of the macaque monkey," Nature Neuroscience 4, 409-416 (2001).

79. J. S. Lund, Q. Wu, P. T. Hadingham, and J. B. Levitt, "Cells and circuits contributing to functional properties in area V1 of macaque monkey cerebral cortex: bases of neuroanatomically realistic models," Journal of Anatomy 187, 563-581 (1995).

80. T. R. Vidyasagar, J. J. Kulikowski, A. Robson, and B. Dreher, "Responses of V1 cells in primate reveal excitatory convergence of $\mathrm{P}$ and $\mathrm{M}$ channels," European Journal of Neuroscience 10, S239 (1998).

81. J. J. Kulikowski and V. Walsh, "Demonstration of binocular fusion of color and texture," in Early Vision and Beyond, T. Papathomas, ed., pp. 27-32 (MIT Press, Cambridge, Massachusetts, 1995).

82. J. J. Kulikowski, "Spatial and temporal properties of chromatic processing: Separation of colour from chromatic pattern mechanisms," in John Dalton's Colour Vision Legacy, pp. 133-146 (Taylor \& Francis Ltd., London, 1997).

83. K. Kranda and P. E. King-Smith, "What can colour thresholds tell us about the nature of the underlying detection mechanisms," Ophthalmic and Physiological Optics 4, 83-87 (1984).

84. C. W. G. Clifford, B. Spehar, S. G. Solomon, P. R. Martin, and Q. Zaidi, "Interactions between color and luminance in the perception of orientation." Journal of Vision 3(2), 106-115 (2003). 


\section{Figure Captions}

Figure 1. Spectral Sensitivity at $1 \mathrm{~Hz}$ as a function of wavelength is plotted for two observers, RB (a) and JK (b). The curves for bars are shifted down by one and two log units, for clarity. Top curves, marked by empty circles, are standard spectral sensitivity functions on a white background $(3350 \mathrm{~K})$ with its characteristic peaks. Intrusion of the luminance mechanism makes the notch shallower as is shown by spectral sensitivity curve on yellow background $(2520 \mathrm{~K})$, marked by filled circles. Bar-like stimuli on a white background reduces spectral sensitivity in the blue range, only slightly for wide bars (empty squares) and more substantial for narrow bars (empty triangles). The $25 \mathrm{~Hz}$ homochromatic flicker function approximating the luminosity function is plotted for a narrow bar on white (dotted line) only for comparison. Spectral sensitivity for bars on yellow background (filled squares and triangles) increases in the blue range, as expected. Error bars are 1SD.

Figure 2. Results for (a) blue $(450 \mathrm{~nm})$ and (b) yellow $(574 \mathrm{~nm})$ spot stimuli on yellow background as a function of Temporal Frequency [Hz]. The measured thresholds (for observer RB, JK and AG) are shown as solid dots and the predicted sensitivity curves for the best-fitting models in each class are shown as lines. The solid line represents model $M_{01}$; the dashed line model $M_{02}$; and the dash-dot line model $M_{012}$. The predicted sensitivity curves for the one-filter model $M_{0}$ gives the least likely fits (for details see third row of Tables 2, 3 and 5,6), and are therfore not shown. The two-filter model $M_{01}$ is the model 
that fits the data for the blue spot best, whereas it is the three-filter model $M_{012}$ that fits the yellow spot best.

Figure 3. Results for blue $(450 \mathrm{~nm})$ and yellow $(574 \mathrm{~nm})$ bar-like stimuli on white or yellow backgrounds as a function of Temporal Frequency [Hz]. Data (for observer RB): the conventions are as in Fig. 2. The two-filter model $M_{01}$ fits the results for the blue bar best, whereas it is the three-filter model $M_{012}$ that fits the yellow bar best.

Figure 4. Results for blue (450 nm) and yellow (574 nm) bar-like stimuli on white or yellow backgrounds as a function of Temporal Frequency [Hz]. Data (for observer JK): the conventions are as in Fig. 2. The two-filter model $M_{01}$ fits the results for the blue bar best, whereas it is the three-filter model $M_{012}$ that fits the yellow bar best.

Figure 5. Results for blue (450 nm) and yellow (574 nm) bar-like stimuli on white or yellow backgrounds as a function of Temporal Frequency [Hz]. Data (for observer AG): the conventions are as in Fig. 2. The two-filter model $M_{01}$ fits the results for the blue bar best, whereas it is the three-filter model $M_{012}$ that fits the yellow bar best. 


\section{Figures}

Fig. 1.
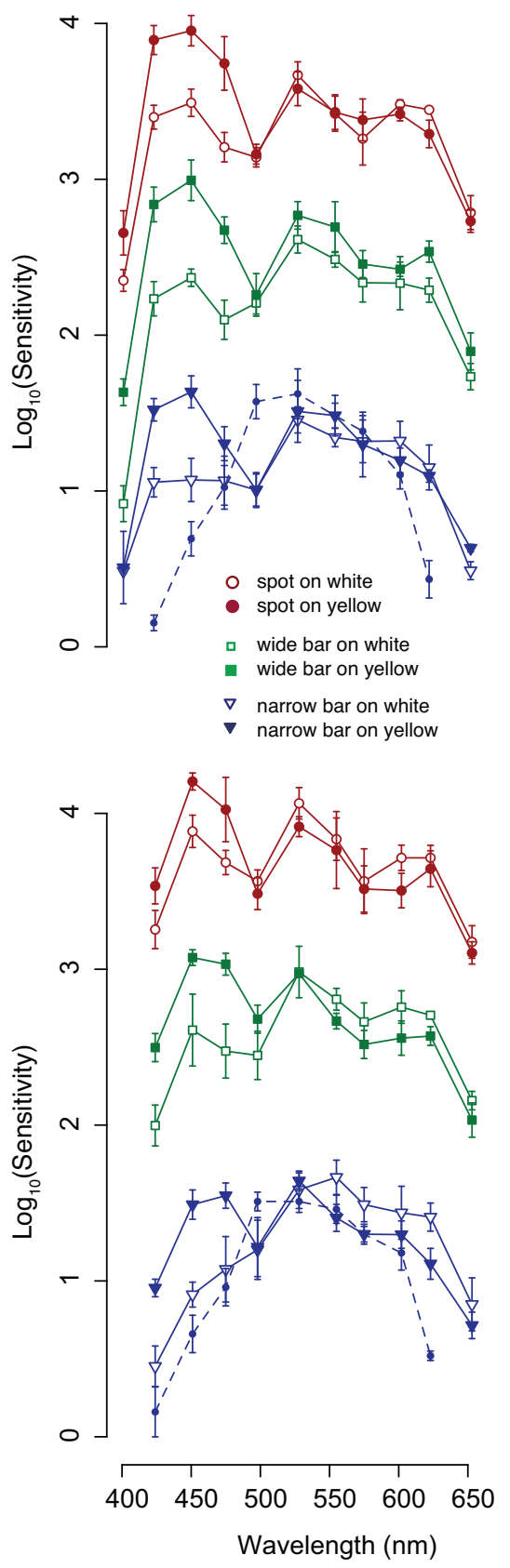
Fig. 2.
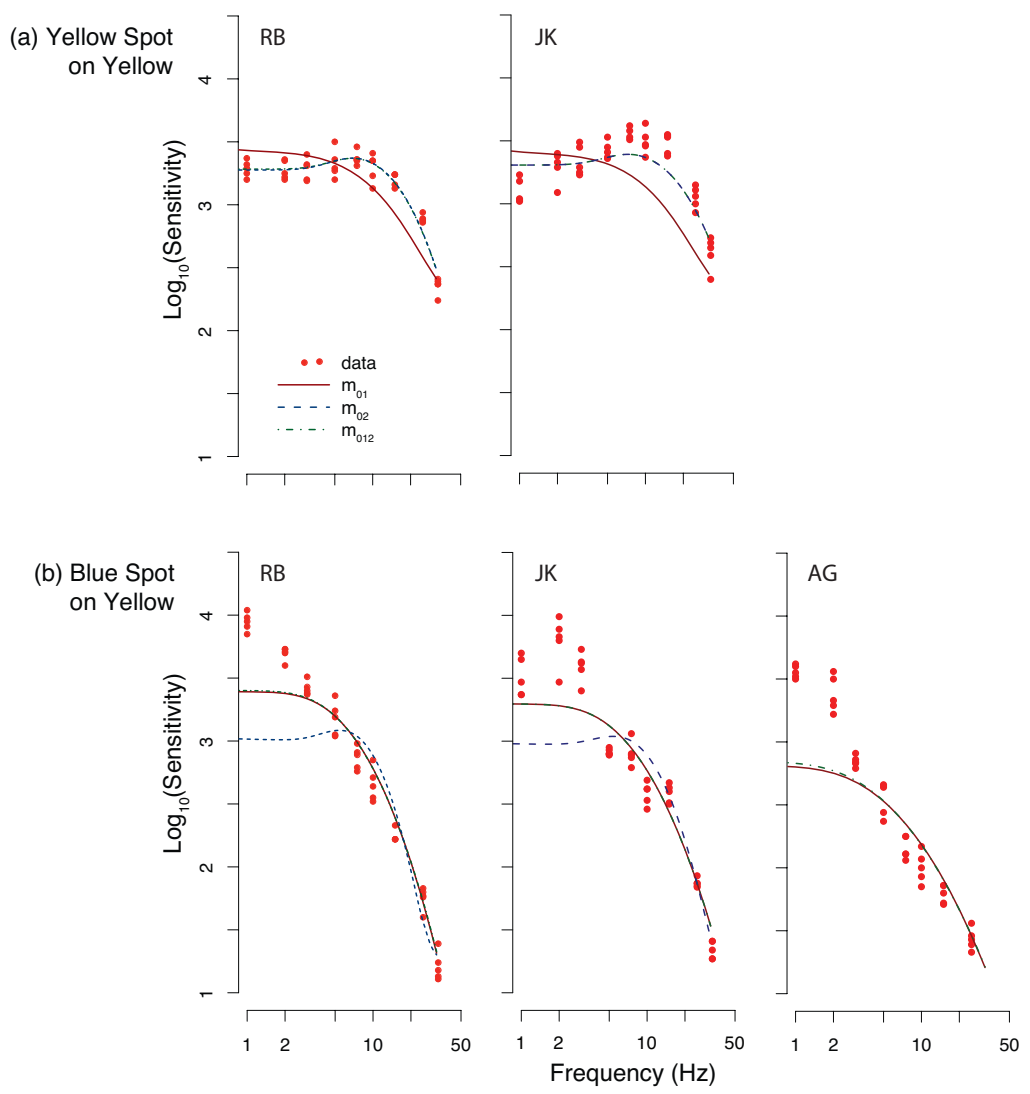
Fig. 3.
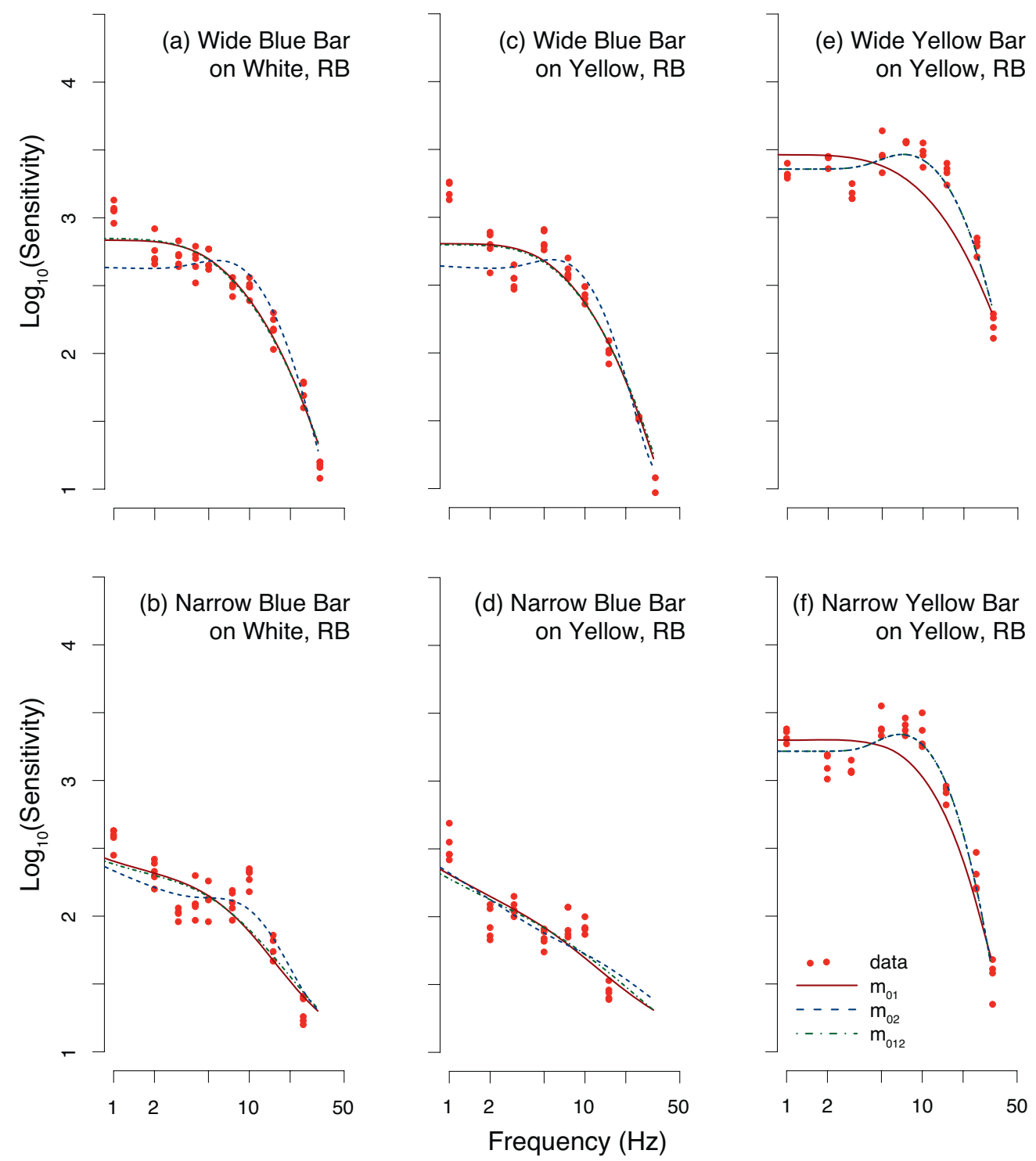
Fig. 4.
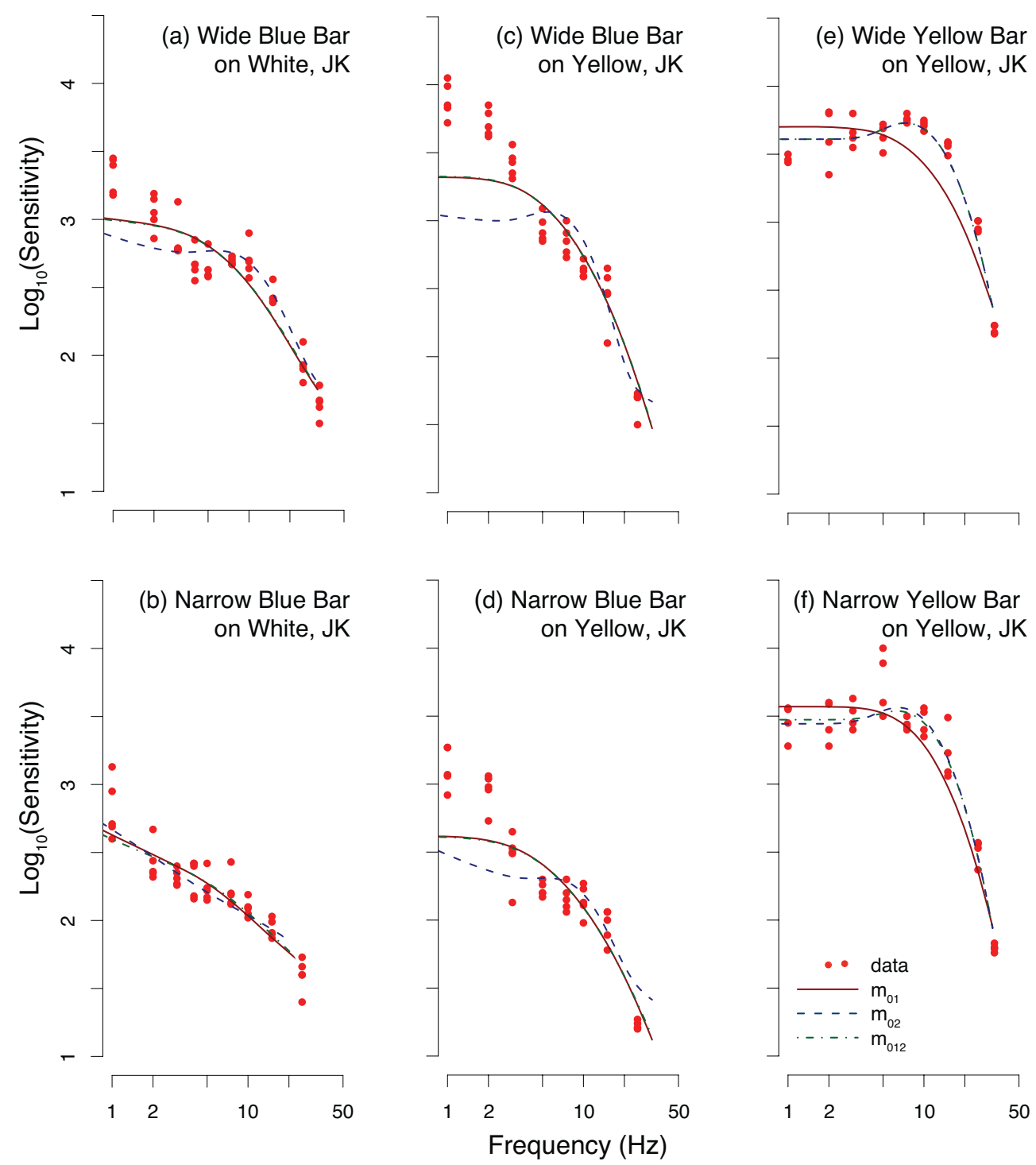
Fig. 5.
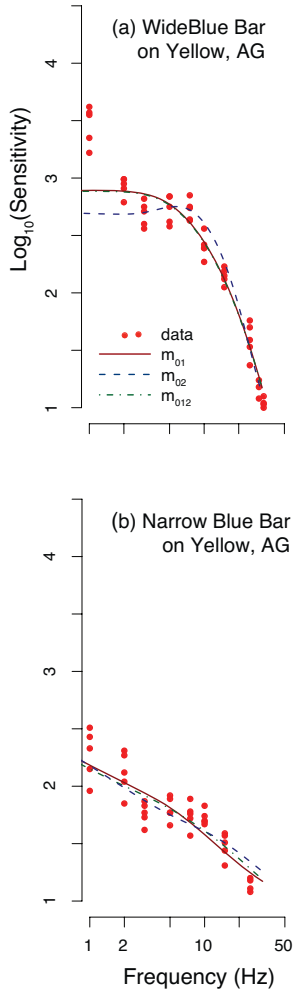


\section{Tables}

Table 1. Parameters of the Gaussian priors on the parameters $\tau$ and $\sigma$. Note that the prior (4) depends on the features $f_{j}(\tau, \sigma)$, which were evaluated numerically.

\begin{tabular}{lcc} 
Feature $f_{j}(\tau, \sigma)$ & $m_{j}$ & $s_{j}$ \\
\hline$f_{0}(\tau, \sigma)$ : freq. at half-maximal gain for the low-pass filter & $8 \mathrm{~Hz}$. & $3 \mathrm{~Hz}$. \\
$f_{1}(\tau, \sigma)$ : freq. at peak gain for the 1st band-pass filter & $4 \mathrm{~Hz}$. & $1 \mathrm{~Hz}$. \\
$f_{2}(\tau, \sigma)$ : freq. at peak gain for the 2nd band-pass filter & $14 \mathrm{~Hz}$. & $3 \mathrm{~Hz}$. \\
\hline
\end{tabular}


Table 2. Results for blue $(450 \mathrm{~nm})$ stimuli on white or yellow backgrounds for observer RB. The total likelihood score of the best model is italicized. The column headed $p_{1}$ gives the $p$-values with regards to whether each of the two-filter or three-filter models is more likely than the one-filter model $M_{0}$. The column headed $p_{2}$ gives the $p$-values with regards to whether each of the two-filter models, $M_{01}$ or $M_{02}$, is more likely than the three-filter model $M_{012}$.

\begin{tabular}{|c|c|c|c|c|c|c|}
\hline Stimulus & Model & Prior & Fit & Total & $p_{1}$ & $p_{2}$ \\
\hline Wide blue bar & $M_{0}$ & -8.26 & -118.55 & -126.81 & & \\
\hline \multirow[t]{3}{*}{ on white } & $M_{01}$ & -8.24 & -70.77 & -79.01 & $<0.01$ & \\
\hline & $M_{02}$ & -13.29 & -74.43 & -87.72 & $<0.01$ & \\
\hline & $M_{012}$ & -8.17 & -70.95 & -79.12 & $<0.01$ & $>0.99$ \\
\hline Narrow blue bar & $M_{0}$ & -8.02 & -71.95 & -79.97 & & \\
\hline \multirow[t]{3}{*}{ on white } & $M_{01}$ & -8.09 & -68.21 & -76.30 & $<0.01$ & \\
\hline & $M_{02}$ & -12.28 & -65.47 & -77.75 & 0.04 & \\
\hline & $M_{012}$ & -8.10 & -68.10 & -76.20 & 0.02 & 0.65 \\
\hline Blue spot & $M_{0}$ & -8.26 & -169.28 & -177.54 & & \\
\hline \multirow[t]{3}{*}{ on yellow } & $M_{01}$ & -14.53 & -73.31 & -87.84 & $<0.01$ & \\
\hline & $M_{02}$ & -16.14 & -95.51 & -111.65 & $<0.01$ & \\
\hline & $M_{012}$ & -14.83 & -73.04 & -87.87 & $<0.01$ & $>0.99$ \\
\hline Wide blue bar & $M_{0}$ & -8.26 & -98.37 & -106.63 & & \\
\hline \multirow[t]{3}{*}{ on yellow } & $M_{01}$ & -8.82 & -59.48 & -68.30 & $<0.01$ & \\
\hline & $M_{02}$ & -14.52 & -61.52 & -76.04 & $<0.01$ & \\
\hline & $M_{012}$ & -8.53 & -59.80 & -68.33 & $<0.01$ & $>0.99$ \\
\hline Narrow blue bar & $M_{0}$ & -8.02 & -53.55 & -61.57 & & \\
\hline \multirow[t]{3}{*}{ on yellow } & $M_{01}$ & -8.02 & -53.32 & -61.34 & 0.50 & \\
\hline & $M_{02}$ & -8.02 & -53.35 & -61.37 & 0.53 & \\
\hline & $M_{012}$ & -8.02 & -53.19 & -61.21 & 0.70 & 0.61 \\
\hline
\end{tabular}


Table 3. Results for blue (450 nm) stimuli on white or yellow backgrounds for observer JK. The conventions are as in Table 2 .

\begin{tabular}{|c|c|c|c|c|c|c|}
\hline Stimulus & Model & Prior & Fit & Total & $p_{1}$ & $p_{2}$ \\
\hline Wide blue bar & $M_{0}$ & -8.10 & -91.99 & -100.09 & & \\
\hline \multirow[t]{3}{*}{ on white } & $M_{01}$ & -8.02 & -76.66 & -84.68 & $<0.01$ & \\
\hline & $M_{02}$ & -12.44 & -77.16 & -89.60 & $<0.01$ & \\
\hline & $M_{012}$ & -8.02 & -76.63 & -84.65 & $<0.01$ & 0.81 \\
\hline Narrow blue bar & $M_{0}$ & -8.02 & -65.58 & -73.60 & & \\
\hline \multirow[t]{3}{*}{ on white } & $M_{01}$ & -8.02 & -64.53 & -72.55 & 0.15 & \\
\hline & $M_{02}$ & -8.05 & -65.50 & -73.55 & 0.75 & \\
\hline & $M_{012}$ & -8.02 & -64.50 & -72.52 & 0.34 & 0.81 \\
\hline Blue spot & $M_{0}$ & -8.26 & -148.16 & -156.42 & & \\
\hline \multirow[t]{3}{*}{ on yellow } & $M_{01}$ & -10.72 & -76.15 & -86.87 & $<0.01$ & \\
\hline & $M_{02}$ & -15.03 & -91.31 & -106.34 & $<0.01$ & \\
\hline & $M_{012}$ & -10.72 & -76.15 & -86.87 & $<0.01$ & $>0.99$ \\
\hline Wide blue bar & $M_{0}$ & -8.26 & -113.93 & -122.19 & & \\
\hline \multirow[t]{3}{*}{ on yellow } & $M_{01}$ & -12.36 & -70.50 & -82.86 & $<0.01$ & \\
\hline & $M_{02}$ & -16.19 & -85.62 & -101.81 & $<0.01$ & \\
\hline & $M_{012}$ & -12.56 & -70.32 & -82.88 & $<0.01$ & $>0.99$ \\
\hline Narrow blue bar & $M_{0}$ & -8.10 & -81.04 & -89.14 & & \\
\hline \multirow[t]{3}{*}{ on yellow } & $M_{01}$ & -8.63 & -66.80 & -75.43 & $<0.01$ & \\
\hline & $M_{02}$ & -13.77 & -73.56 & -87.33 & 0.06 & \\
\hline & $M_{012}$ & -8.53 & -66.97 & -75.50 & $<0.01$ & $>0.99$ \\
\hline
\end{tabular}


Table 4. Results for blue (450 nm) stimuli on white or yellow backgrounds for observer AG. The conventions are as in Tabel 2 .

\begin{tabular}{|c|c|c|c|c|c|c|}
\hline Stimulus & Model & Prior & Fit & Total & $p_{1}$ & $p_{2}$ \\
\hline Blue spot & $M_{0}$ & -8.26 & -95.31 & -103.57 & & \\
\hline \multirow[t]{3}{*}{ on yellow } & $M_{01}$ & -11.18 & -77.37 & -88.55 & $<0.01$ & \\
\hline & $M_{02}$ & -8.26 & -95.31 & -103.57 & $>0.99$ & \\
\hline & $M_{012}$ & -12.33 & -76.24 & -88.57 & $<0.01$ & $>0.99$ \\
\hline Wide blue bar & $M_{0}$ & -8.26 & -146.22 & -154.48 & & \\
\hline \multirow[t]{3}{*}{ on yellow } & $M_{01}$ & -9.76 & -76.34 & -86.10 & $<0.01$ & \\
\hline & $M_{02}$ & -14.57 & -81.79 & -96.36 & $<0.01$ & \\
\hline & $M_{012}$ & -9.76 & -76.36 & -86.12 & $<0.01$ & $>0.99$ \\
\hline Narrow blue bar & $M_{0}$ & -8.02 & -58.64 & -66.66 & & \\
\hline \multirow[t]{3}{*}{ on yellow } & $M_{01}$ & -8.02 & -58.02 & -66.04 & 0.27 & \\
\hline & $M_{02}$ & -8.02 & -58.23 & -66.25 & 0.37 & \\
\hline & $M_{012}$ & -8.02 & -57.74 & -65.76 & 0.41 & 0.45 \\
\hline
\end{tabular}


Table 5. Results for yellow $(574 \mathrm{~nm})$ stimuli on yellow background for observer RB. The conventions are as in Table 2 . The three-filter model $M_{012}$ has a small numerical edge over the simpler models in two out of three conditions, but is not significantly different from the two-filter model $M_{02}$.

\begin{tabular}{|c|c|c|c|c|c|c|}
\hline Stimulus & Model & Prior & Fit & Total & $p_{1}$ & $p_{2}$ \\
\hline Yellow spot & $M_{0}$ & -10.14 & -100.88 & -111.02 & & \\
\hline \multirow[t]{3}{*}{ on yellow } & $M_{01}$ & -10.90 & -75.64 & -86.54 & $<0.01$ & \\
\hline & $M_{02}$ & -10.75 & -62.31 & -73.06 & $<0.01$ & \\
\hline & $M_{012}$ & -10.75 & -62.30 & -73.05 & $<0.01$ & 0.89 \\
\hline Wide yellow bar & $M_{0}$ & -8.52 & -107.09 & -115.61 & & \\
\hline \multirow[t]{3}{*}{ on yellow } & $M_{01}$ & -10.14 & -68.53 & -78.67 & $<0.01$ & \\
\hline & $M_{02}$ & -12.19 & -52.62 & -64.81 & $<0.01$ & \\
\hline & $M_{012}$ & -12.19 & -52.60 & -64.79 & $<0.01$ & 0.84 \\
\hline Narrow yellow bar & $M_{0}$ & -9.05 & -178.20 & -187.25 & & \\
\hline \multirow[t]{3}{*}{ on yellow } & $M_{01}$ & -12.52 & -65.58 & -78.10 & $<0.01$ & \\
\hline & $M_{02}$ & -13.82 & -53.36 & -67.18 & $<0.01$ & \\
\hline & $M_{012}$ & -13.82 & -53.36 & -67.18 & $<0.01$ & $>0.99$ \\
\hline
\end{tabular}


Table 6. Results for yellow $(574 \mathrm{~nm})$ stimuli on yellow background for observer JK. The conventions are as in Table 2.

\begin{tabular}{|c|c|c|c|c|c|c|}
\hline Stimulus & Model & Prior & Fit & Total & $p_{1}$ & $p_{2}$ \\
\hline Yellow spot & $M_{0}$ & -11.11 & -121.52 & -132.63 & & \\
\hline \multirow[t]{3}{*}{ on yellow } & $M_{01}$ & -13.80 & -104.08 & -117.88 & $<0.01$ & \\
\hline & $M_{02}$ & -9.61 & -72.44 & -82.05 & $<0.01$ & \\
\hline & $M_{012}$ & -9.61 & -72.45 & -82.06 & $<0.01$ & $>0.99$ \\
\hline Wide yellow bar & $M_{0}$ & -9.25 & -138.16 & -147.41 & & \\
\hline \multirow[t]{3}{*}{ on yellow } & $M_{01}$ & -10.86 & -66.87 & -77.73 & $<0.01$ & \\
\hline & $M_{02}$ & -13.43 & -51.35 & -64.78 & $<0.01$ & \\
\hline & $M_{012}$ & -13.43 & -51.35 & -64.78 & $<0.01$ & $>0.99$ \\
\hline Narrow yellow bar & $M_{0}$ & -9.05 & -170.64 & -179.69 & & \\
\hline \multirow[t]{3}{*}{ on yellow } & $M_{01}$ & -11.96 & -60.53 & -72.49 & $<0.01$ & \\
\hline & $M_{02}$ & -13.73 & -54.41 & -68.14 & $<0.01$ & \\
\hline & $M_{012}$ & -13.51 & -54.31 & -67.82 & $<0.01$ & 0.42 \\
\hline
\end{tabular}

